\title{
Prevalence, awareness, and control of hypertension among Bangladeshi adults: an analysis of demographic and health survey 2017-18
}

\author{
Gulam Muhammed Al Kibria ${ }^{1 *}$ (D) Rajat Das Gupta ${ }^{2}$ (D) and Jannatun Nayeem ${ }^{3}$
}

\begin{abstract}
Background: The prevalence of hypertension is increasing in Bangladesh, however, few recent studies investigated the proportion of people and factors associated with prevalence, awareness, and control of this condition in this country. This study investigated these among Bangladeshi adults.

Methods: Using Bangladesh Demographic and Health Survey 2017-18 data, a cross-sectional study was conducted. Multilevel logistic regression analysis was employed after descriptive analysis and prevalence estimation.

Results: Among 12,926 persons (mean age: 40 years, 57\% women), the prevalence of hypertension was $27.4 \%$ ( $n=$ 3551), it was 28.4 and $26.2 \%$ among females and males, respectively. Among hypertensive people, about $42.4 \%$ ( $n=1508$ ) people were aware of having it, $48.7 \%$ among females and $33.5 \%$ among males. Of the 1313 people who were taking antihypertensive medication, only 33.8\% $(n=443)$ had controlled hypertension, 34.7 and $31.7 \%$ among females and males, respectively.

Among the studied factors associated with hypertension, people with older age, female gender, overweight/ obesity, diabetes, richer wealth quintiles, and residence in some administrative divisions had higher odds of hypertension $(p<0.05)$. However, the odds of awareness was lower among younger people, males, and people without overweight/obesity, diabetes, or richer wealth quintiles. Odds of controlled hypertension was also lower among people with older age and higher among college-educated people.

Conclusion: This study identified several important factors associated with prevalence, awareness, and control of hypertension. It is important to address these factors with nationwide prevention and control programs.
\end{abstract}

Keywords: Hypertension, Awareness, Prevalence, Control, Determinants, Risk factors

\footnotetext{
*Correspondence: gkibria1@outlook.com

'Department of Epidemiology and Public Health, University of Maryland

School of Medicine, Baltimore, MD 21201, USA

Full list of author information is available at the end of the article
}

(C) The Author(s). 2021 Open Access This article is licensed under a Creative Commons Attribution 4.0 International License, which permits use, sharing, adaptation, distribution and reproduction in any medium or format, as long as you give appropriate credit to the original author(s) and the source, provide a link to the Creative Commons licence, and indicate if changes were made. The images or other third party material in this article are included in the article's Creative Commons licence, unless indicated otherwise in a credit line to the material. If material is not included in the article's Creative Commons licence and your intended use is not permitted by statutory regulation or exceeds the permitted use, you will need to obtain permission directly from the copyright holder. To view a copy of this licence, visit http://creativecommons.org/licenses/by/4.0/ The Creative Commons Public Domain Dedication waiver (http://creativecommons.org/publicdomain/zero/1.0/) applies to the data made available in this article, unless otherwise stated in a credit line to the data. 


\section{Background}

Hypertension is a leading risk factor for cardiovascular disease and a major cause of deaths and disabilities among adults worldwide [1, 2]. Recent trends suggest that the prevalence of hypertension is increasing at an alarming rate among people in low- and middle-income countries (LMICs) [2]. Bangladesh, an LMIC in South Asia, is of no exception from this rising trend [3, 4]. According to the latest Bangladesh Demographic and Health Survey (BDHS) 2017-18, a nationally representative survey from Bangladesh, about $45 \%$ of the women and $34 \%$ of the men aged 35 years and older are hypertensive in this country [4]. This is a significant rise from the previous estimates of 32 and $20 \%$ among women and men of same age group in 2011, respectively [4, 5]. The ongoing demographic transition with an increasing proportion of aging population along with changes in socioeconomic status, lifestyles, and dietary habits of Bangladeshi people could contribute to this higher prevalence [2, 4-6]. This report also shows that more than half of the hypertensive people are not aware of their hypertensive condition [4].

It is important to halt this continues rise in prevalence of hypertension in this country. In addition, to reduce future burden of complications resulting from hypertension, increasing the awareness of hypertension among those who are hypertensive and increasing the control of blood pressure (BP) among those who take any antihypertensive drugs are important [2, 7]. Therefore, identifying the determinants or associated factors of prevalence, awareness, and control of hypertension and addressing them with prevention programs are critical. Using BDHS 2011 data, multiple previous studies reported that people with older age, female gender, urban residence, and higher education level or wealth status had higher prevalence of hypertension [3, 6, 8-10]. These factors may also contribute to awareness and control $[7,11]$. However, the proportion of people with prevalence, awareness, or control of hypertension according to background characteristics and factors associated with these three outcomes in recent years are not known. In this study, using a nationally representative sample of Bangladeshi adults, we attempted to estimate prevalence, awareness, and control of hypertension along with the factors that influence these three outcomes.

\section{Methods}

\section{Study design and setting}

We conducted a cross-sectional study by performing secondary analysis on BDHS 2017-18 data. This is the eighth nationwide survey to report major demographic and health indicators on the country. Data collection took place from October 2017 to March 2018. Mitra and Associates, a private research firm in Bangladesh, implemented it [4]. We analyzed the data in January 2021.
A two-stage stratified sample of households was selected. First, for the sampling frame, a list of enumeration areas (EAs) was prepared from the 2011 Population and Housing Census of the People's Republic of Bangladesh, 250 and 425 EAs from urban and rural areas, respectively. EA was the primary sampling unit and was selected with probability proportional to size. In the EA, a complete list of all the households were done. Then, in the second stage, an average of 30 households were selected from each EA. A total of 20,250 households were selected in this way. From these households, one-fourth of the households were randomly selected for $\mathrm{BP}$ and fasting plasma glucose measurements. All adults (i.e., 18 years of age or older) living in these households were invited to participate for measuring BP and fasting plasma glucose. Among 14,704 eligible women and men, about $90 \%$ participated. The quality of data collection was monitored by 4 quality control teams from the data collection organization. The questionnaires were translated and validated in the context of Bangladesh. Details of BDHS 2017-18, including survey design, methodologies, sample size calculation, questionnaires, and other statistics are available elsewhere [4].

\section{Outcome variables}

The outcome measures of this study were 'prevalence (i.e., presence of hypertension) of hypertension', 'awareness of having hypertension' and 'controlled hypertension among those who take antihypertensive drug'. Systolic BP (SBP) and diastolic BP (DBP) were measured for three times (in millimeters of mercury [ $\mathrm{mmHg}]$ ) with an interval of five minutes between measurements. BP was recorded by digital oscillometric BP device (i.e., LIFE SOURCE ${ }^{\circledast}$ UA-767 Plus BP monitor). Appropriate cuff sizes were used [4]. The mean of the last two measurements was used to classify a respondent as hypertensive. If the third measurement was not available, then the second measurement was used. In case of missing second and third measurements, the first measurement was used. Similar to BDHS 2017-18, we used World Health Organization - International Society of Hypertension cutoffs to classify a person as hypertensive [4, 12]. Therefore, a person with an SBP/DBP of $140 / 90 \mathrm{mmHg}$ or more was categorized as hypertensive. In addition, participants who reported that they were taking any BP lowering drugs were considered as hypertensive [12].

Among those we found hypertensive, we considered a person as aware if the person knew the diagnosis of hypertension from a doctor or nurse (i.e., "Have you ever been told by a doctor or other health worker that you have high BP or hypertension?") [4]. Among those who were taking any antihypertensive drugs, we considered 'controlled hypertension' if the person had the SBP/DBP below 140/90 $\mathrm{mmHg}$ during the measurement of BP. 


\section{Potential determinants/independent variables}

Based on published reports and data structure, we investigated age, gender, overweight/obesity, diabetes, education level, wealth quintile, and place and division of residence as potential determinants. Age was categorized into $18-34,35-44,45-54,55-64$, and 65 or above years. Overweight/obesity was obtained from body mass index (BMI). BMI was obtained by dividing weight (in $\mathrm{Kg}$ ) with height (in $\mathrm{m}^{2}$ ). Body weight was measured using a lightweight, electronic SECA 878 scale screen. Height was obtained with a ShorrBoard ${ }^{\circ}$ measuring board in standing position [4]. Persons with a BMI $23 \mathrm{Kg} / \mathrm{m}^{2}$ or more were classified as having overweight/obesity [13, 14]. Fasting plasma glucose was measured using HemoCue 201 RT analyzer [4]. We classified a participant as diabetic if the fasting plasma glucose was $7 \mathrm{mmol} / \mathrm{l}$ or more. People who were taking antidiabetic drugs were also considered as diabetic. Participants reported their gender (i.e., male or female) and place (i.e., rural or urban) and division (i.e., Barishal, Chattagram, Dhaka, Khulna, Mymensingh, Rajshahi, Rangpur, or Sylhet) of residence. Education level was categorized into no formal education, primary (i.e., 1-5 school years), secondary (i.e., 6-10 school years), and college or above (i.e., 11 or more school years). Wealth status was obtained from household belongings and basic household construction materials. Similar to previous BDHS, principal component analysis was used to obtain a wealth index score from those components and was then stratified into quintiles: poorest, poorer, middle, richer, and richest $[4,15]$.

\section{Statistical analysis}

First, we described the study sample with weighted numbers and percentages for categorical variables and means and standard errors (SE) for continuous variables according to the prevalence, awareness, and control status (Tables 1, 2, and 3). To compare continuous variables, we used t-tests and to compare categorical variables, we used chi-square tests. We also reported the prevalence, awareness, and controlled hypertension according to the study variables (Table 4). We have also reported the prevalence among people 30 years of age (Supplemental Table 1). We then obtained the unadjusted odds ratios (UOR) along with 95\% confidence intervals (CI) using multilevel logistic regression (Supplemental Table 2). Multilevel regression was done due to the hierarchical nature of the BDHS data (Table 5). Variables with a significance level (i.e., $p$-values) up to 0.2 were investigated into multivariable models to obtain the adjusted OR (AOR) [16]. We obtained variance inflation factors (VIF) with a fake linear regression model to check multicollinearity. We used the provided sample weights to obtain all estimates [4]. Stata 14.0 (College Station, TX, USA) was used to analyze data [17].

\section{Ethical approval}

The Institutional Review Boards (IRBs) of the ICF International, Rockville, Maryland, USA and Bangladesh Medical Research Council, Dhaka, Bangladesh approved the BDHS 2017-18 protocol. The permission for using the data was obtained in December 2020. All participants provided informed consent to participate into the survey.

\section{Results}

A total of 12,926 participants were included in the analysis (Table 1). Mean SBP and DBP were $122.3 \mathrm{mmHg}$ (SE: 0.25 ) and $80.2 \mathrm{mmHg}$ (SE: 0.14), respectively. The average age of the participants was 39.6 years (SE: 0.15) and about $56.8 \%(n=7343)$ of the participants were females; hypertensive people had a higher proportion of older people or females than people without hypertension. About 9.9\% $(n=1199)$ and $40.2 \%(n=5129)$ of the participants had diabetes and overweight/obesity, respectively. About $26.6 \%$ of the participants had no formal education or $27.4 \%$ of the participants were living in urban areas. The overall proportion of people with diabetes, overweight/obesity, or urban residence was higher among people with hypertension than those without hypertension. About one-fourth of the participants were from Dhaka division, 23.9\% $(n=3096)$.

Among people who were hypertensive $(n=3551)$, the mean SBP and DBP were $145.8 \mathrm{mmHg}$ (SE: 0.43 ) and $91.5 \mathrm{mmHg}$ (SE: 0.21), respectively (Table 2). The mean SBP, DBP, age, fasting plasma glucose, and BMI of people with awareness was higher than those without awareness. The proportion of male participants was higher among those without awareness compared to those with it, $47.6 \%(n=973)$ and $32.5 \%(n=489)$, respectively. The awareness level also differs by wealth status and place or division of residence.

Among those who were taking antihypertensive drugs $(n=1313)$, the mean age and fasting plasma glucose was higher among those who had uncontrolled BP $(n=870)$ than those who had controlled BP $(n=443)$ (Table 3). Among all those who were taking antihypertensive, more than two-thirds were females, $68.0 \%(n=893)$, it was similar among those with or without controlled hypertension. About one-fourth had diabetes and two-third had overweight/obesity, 23.6\% $(n=293)$ and $62.2 \%(n=801)$, respectively. Controlled hypertension level did not differ by wealth status or place of residence, however, it differed by education level and division of residence $(p<0.05)$.

Table 4 shows the prevalence, awareness, and control of hypertension according to background characteristics. The overall prevalence $(95 \% \mathrm{CI})$ of hypertension was $27.4 \%$ (26.5 to $28.5 \%$ ), it increased with age, $12.7 \%$ (11.8 to $13.8 \%$ ), $28.4 \%$ (26.6 to $30.3 \%$ ), $38.5 \%$ (35.6 to $41.4 \%$ ), 
Table 1 Distribution of the study sample according to presence of hypertension

\begin{tabular}{|c|c|c|c|c|}
\hline \multirow[t]{2}{*}{ Variables } & \multirow{2}{*}{$\begin{array}{l}\text { Total } \\
(n=12,926)\end{array}$} & \multicolumn{3}{|l|}{ Hypertension } \\
\hline & & Yes $(n=3551)$ & No $(n=9375)$ & $p$-value \\
\hline Mean systolic BP (SE), mmHg & $122.3(0.25)$ & $145.8(0.42)$ & $113.5(0.15)$ & $<0.001$ \\
\hline Mean diastolic BP (SE), mmHg & $80.2(0.14)$ & $91.5(0.21)$ & $76.0(0.11)$ & $<0.001$ \\
\hline \multicolumn{5}{|l|}{ Age (in years) } \\
\hline Mean (SE) & $39.6(0.15)$ & $49.0(0.30)$ & $36.2(0.17)$ & $<0.001$ \\
\hline 18 to 34 & $5787(44.8)$ & $738(20.8)$ & $5049(53.9)$ & $<0.001$ \\
\hline 35 to 44 & $2585(20)$ & $735(20.7)$ & $1850(19.7)$ & \\
\hline 45 to 54 & $1815(14)$ & $698(19.7)$ & $1116(11.9)$ & \\
\hline 55 to 64 & $1454(11.3)$ & $680(19.1)$ & $775(8.3)$ & \\
\hline 65 or more & $1285(9.9)$ & $700(19.7)$ & $585(6.2)$ & \\
\hline \multicolumn{5}{|l|}{ Gender } \\
\hline Female & $7343(56.8)$ & $2088(58.8)$ & $5255(56.1)$ & 0.01 \\
\hline Male & $5583(43.2)$ & $1463(41.2)$ & $4120(43.9)$ & \\
\hline \multicolumn{5}{|l|}{ Diabetes } \\
\hline Mean fasting plasma glucose (SE), mmol/L & $5.7(0.02)$ & $6.0(0.05)$ & $5.6(0.02)$ & $<0.001$ \\
\hline No & $10,893(90.1)$ & $2786(83.4)$ & 8107 (92.6) & $<0.001$ \\
\hline Yes & $1199(9.9)$ & $553(16.6)$ & $646(7.4)$ & \\
\hline \multicolumn{5}{|l|}{ Overweight/Obesity } \\
\hline Mean body mass index (SE), $\mathrm{Kg} / \mathrm{m}^{2}$ & $22.4(0.06)$ & $23.7(0.09)$ & $21.9(0.06)$ & $<0.001$ \\
\hline No & $7640(59.8)$ & $1573(45.2)$ & $6067(65.3)$ & $<0.001$ \\
\hline Yes & $5129(40.2)$ & $1904(54.8)$ & $3225(34.7)$ & \\
\hline \multicolumn{5}{|l|}{ Education level } \\
\hline No formal education & $3444(26.6)$ & $1211(34.1)$ & $2233(23.8)$ & $<0.001$ \\
\hline Primary & $3839(29.7)$ & $1047(29.5)$ & $2792(29.8)$ & \\
\hline Secondary & $3790(29.3)$ & $877(24.7)$ & $2914(31.1)$ & \\
\hline College or above & $1853(14.3)$ & $416(11.7)$ & $1437(15.3)$ & \\
\hline \multicolumn{5}{|l|}{ Wealth quintile } \\
\hline Poorest & $2459(19.0)$ & $584(16.4)$ & $1875(20)$ & $<0.001$ \\
\hline Poorer & $2534(19.6)$ & $638(18)$ & $1897(20.2)$ & \\
\hline Middle & $2635(20.4)$ & $705(19.8)$ & $1930(20.6)$ & \\
\hline Richer & $2560(19.8)$ & $723(20.4)$ & $1837(19.6)$ & \\
\hline Richest & $2738(21.2)$ & $902(25.4)$ & $1836(19.6)$ & \\
\hline \multicolumn{5}{|l|}{ Place of residence } \\
\hline Urban & $3542(27.4)$ & $1009(28.4)$ & $2533(27)$ & 0.19 \\
\hline Rural & $9383(72.6)$ & $2542(71.6)$ & $6842(73)$ & \\
\hline \multicolumn{5}{|l|}{ Division of residence } \\
\hline Dhaka & $3096(23.9)$ & $730(20.5)$ & $2366(25.2)$ & $<0.001$ \\
\hline Barishal & $718(5.6)$ & $234(6.6)$ & $484(5.2)$ & \\
\hline Chattagram & $2237(17.3)$ & $666(18.8)$ & $1571(16.8)$ & \\
\hline Khulna & $1586(12.3)$ & $474(13.3)$ & $1113(11.9)$ & \\
\hline Mymensingh & $1067(8.3)$ & $252(7.1)$ & $815(8.7)$ & \\
\hline Rajshahi & $1829(14.2)$ & $503(14.2)$ & $1326(14.1)$ & \\
\hline Rangpur & $1552(12)$ & $473(13.3)$ & 1079 (11.5) & \\
\hline Sylhet & $840(6.5)$ & $220(6.2)$ & $621(6.6)$ & \\
\hline
\end{tabular}


Table 2 Description of study sample according to awareness among those with hypertension

\begin{tabular}{|c|c|c|c|c|}
\hline \multirow[t]{2}{*}{ Variables } & \multirow{2}{*}{$\begin{array}{l}\text { Total } \\
(n=3551)\end{array}$} & \multicolumn{3}{|l|}{ Awareness } \\
\hline & & Yes $(n=1508)$ & No $(n=2043)$ & $p$-value \\
\hline Mean Systolic BP (SE), mmHg & $145.8(0.43)$ & $148.0(0.77)$ & $144.0(0.42)$ & $<0.001$ \\
\hline Mean diastolic BP (SE), mmHg & $91.5(0.21)$ & $90.8(0.37)$ & $92.0(0.21)$ & 0.004 \\
\hline \multicolumn{5}{|l|}{ Age (in years) } \\
\hline Mean (SE) & $49.0(0.30)$ & $52.1(0.41)$ & $46.8(0.41)$ & $<0.001$ \\
\hline 18 to 34 & $738(20.8)$ & $214(14.2)$ & $524(25.6)$ & $<0.001$ \\
\hline 35 to 44 & $735(20.7)$ & $275(18.3)$ & $460(22.5)$ & \\
\hline 45 to 54 & $698(19.7)$ & $320(21.2)$ & $379(18.5)$ & \\
\hline 55 to 64 & $680(19.1)$ & $361(23.9)$ & $319(15.6)$ & \\
\hline 65 or more & $700(19.7)$ & $338(22.4)$ & $362(17.7)$ & \\
\hline \multicolumn{5}{|l|}{ Gender } \\
\hline Female & $2088(58.8)$ & $1018(67.5)$ & $1070(52.4)$ & $<0.001$ \\
\hline Male & $1463(41.2)$ & $490(32.5)$ & $973(47.6)$ & \\
\hline \multicolumn{5}{|l|}{ Diabetes } \\
\hline Mean fasting plasma glucose (SE), mmol/L & $6.0(0.05)$ & $6.2(0.07)$ & $5.9(0.06)$ & $<0.001$ \\
\hline No & $2786(83.4)$ & $1104(77.7)$ & $1682(87.7)$ & $<0.001$ \\
\hline Yes & $553(16.6)$ & $316(22.3)$ & $237(12.3)$ & \\
\hline \multicolumn{5}{|l|}{ Overweight/Obesity } \\
\hline Mean body mass index (SE), $\mathrm{Kg} / \mathrm{m}^{2}$ & $23.7(0.09)$ & $24.4(0.13)$ & $23.2(0.12)$ & $<0.001$ \\
\hline No & $1573(45.2)$ & $572(38.7)$ & $1001(50.1)$ & $<0.001$ \\
\hline Yes & $1904(54.8)$ & $905(61.3)$ & $999(49.9)$ & \\
\hline \multicolumn{5}{|l|}{ Education level } \\
\hline No Formal Education & $1211(34.1)$ & $524(34.7)$ & $687(33.6)$ & 0.27 \\
\hline Primary & $1047(29.5)$ & $456(30.3)$ & $591(28.9)$ & \\
\hline Secondary & $877(24.7)$ & $369(24.5)$ & $507(24.8)$ & \\
\hline College or above & $416(11.7)$ & $158(10.5)$ & $258(12.6)$ & \\
\hline \multicolumn{5}{|l|}{ Wealth Quintile } \\
\hline Poorest & $584(16.4)$ & $190(12.6)$ & $394(19.3)$ & $<0.001$ \\
\hline Poorer & $638(18.0)$ & $232(15.4)$ & $406(19.8)$ & \\
\hline Middle & $705(19.8)$ & $295(19.6)$ & $410(20.0)$ & \\
\hline Richer & $723(20.4)$ & $333(22.1)$ & $390(19.1)$ & \\
\hline Richest & $902(25.4)$ & $458(30.4)$ & $444(21.7)$ & \\
\hline \multicolumn{5}{|l|}{ Place of residence } \\
\hline Urban & $1009(28.4)$ & $470(31.2)$ & $539(26.4)$ & 0.006 \\
\hline Rural & $2542(71.6)$ & 1037 (68.8) & 1505 (73.6) & \\
\hline \multicolumn{5}{|l|}{ Division of Residence } \\
\hline Dhaka & $730(20.5)$ & $332(22.0)$ & $398(19.5)$ & 0.005 \\
\hline Barishal & $234(6.6)$ & $104(6.9)$ & $130(6.4)$ & \\
\hline Chattagram & $666(18.8)$ & $296(19.7)$ & $370(18.1)$ & \\
\hline Khulna & $474(13.3)$ & $208(13.8)$ & $266(13.0)$ & \\
\hline Mymensingh & $252(7.1)$ & $107(7.1)$ & $145(7.1)$ & \\
\hline Rajshahi & $503(14.2)$ & $196(13.0)$ & $307(15.0)$ & \\
\hline Rangpur & $473(13.3)$ & $157(10.4)$ & $316(15.5)$ & \\
\hline Sylhet & $220(6.2)$ & $107(7.1)$ & $112(5.5)$ & \\
\hline
\end{tabular}

Number are presented along with column percentage unless otherwise specified 
Table 3 Controlled hypertension status among those taking antihypertensive drugs

\begin{tabular}{|c|c|c|c|c|}
\hline \multirow[t]{2}{*}{ Variables } & \multirow{2}{*}{$\begin{array}{l}\text { Overall } \\
(n=1313)\end{array}$} & \multicolumn{3}{|c|}{ Controlled Hypertension } \\
\hline & & Yes $(n=443)$ & No $(n=870)$ & $p$-values \\
\hline Mean systolic BP (SE), mmHg & $147.1(0.82)$ & $121.7(0.57)$ & $160.0(0.83)$ & $<0.001$ \\
\hline Mean diastolic BP (SE), mmHg & $89.8(0.39)$ & $79.3(0.39)$ & $95.2(0.41)$ & $<0.001$ \\
\hline \multicolumn{5}{|l|}{ Age (in years) } \\
\hline Mean (SE) & $52.7(0.42)$ & $48.7(0.75)$ & $54.7(0.51)$ & $<0.001$ \\
\hline 18 to 34 & $163(12.4)$ & $88(19.8)$ & $75(8.6)$ & $<0.001$ \\
\hline 35 to 44 & $236(17.9)$ & $90(20.3)$ & $145(16.7)$ & \\
\hline 45 to 54 & $287(21.9)$ & $91(20.5)$ & $196(22.5)$ & \\
\hline 55 to 64 & $328(25.0)$ & $109(24.6)$ & $219(25.1)$ & \\
\hline 65 or more & $300(22.8)$ & $65(14.7)$ & $235(27.0)$ & \\
\hline \multicolumn{5}{|l|}{ Gender } \\
\hline Female & $893(68.0)$ & $310(70.0)$ & $583(67.0)$ & 0.32 \\
\hline Male & $420(32.0)$ & $133(30.0)$ & $287(33.0)$ & \\
\hline \multicolumn{5}{|l|}{ Diabetes } \\
\hline Mean fasting plasma glucose (SE), $\mathrm{mmol} / \mathrm{L}$ & $6.3(0.08)$ & $5.9(0.11)$ & $6.4(0.10)$ & 0.001 \\
\hline No & $948(76.4)$ & $330(80.1)$ & $618(74.6)$ & 0.043 \\
\hline Yes & $293(23.6)$ & $82(19.9)$ & $211(25.4)$ & \\
\hline \multicolumn{5}{|l|}{ Overweight/Obesity } \\
\hline Mean body mass index (SE), $\mathrm{kg} / \mathrm{m}^{2}$ & $24.4(0.13)$ & $24.5(0.22)$ & $24.4(0.16)$ & 0.69 \\
\hline No & $486(37.8)$ & $168(38.6)$ & $318(37.3)$ & 0.67 \\
\hline Yes & $801(62.2)$ & $268(61.4)$ & $533(62.7)$ & \\
\hline \multicolumn{5}{|l|}{ Education level } \\
\hline No formal education & $468(35.6)$ & $129(29.2)$ & $338(38.9)$ & 0.007 \\
\hline Primary & $395(30.1)$ & $136(30.8)$ & $258(29.7)$ & \\
\hline Secondary & $320(24.3)$ & $122(27.6)$ & $197(22.7)$ & \\
\hline College or above & $131(10.0)$ & $55(12.5)$ & $76(8.7)$ & \\
\hline \multicolumn{5}{|l|}{ Wealth quintile } \\
\hline Poorest & $162(12.4)$ & $52(11.7)$ & $110(12.7)$ & 0.66 \\
\hline Poorer & $193(14.7)$ & $63(14.3)$ & $129(14.9)$ & \\
\hline Middle & $263(20.0)$ & $84(18.9)$ & $179(20.6)$ & \\
\hline Richer & $289(22.0)$ & $110(24.7)$ & $179(20.6)$ & \\
\hline Richest & $407(31.0)$ & $135(30.4)$ & $272(31.3)$ & \\
\hline \multicolumn{5}{|l|}{ Place of residence } \\
\hline Urban & $417(31.8)$ & $150(33.9)$ & $267(30.7)$ & 0.28 \\
\hline Rural & $896(68.2)$ & $293(66.1)$ & $603(69.3)$ & \\
\hline \multicolumn{5}{|l|}{ Division of residence } \\
\hline Dhaka & $297(22.6)$ & $98(22.1)$ & $199(22.9)$ & $<0.001$ \\
\hline Barishal & $92(7.0)$ & $32(7.2)$ & $60(6.8)$ & \\
\hline Chattagram & $273(20.8)$ & $114(25.7)$ & $159(18.3)$ & \\
\hline Khulna & $173(13.2)$ & $44(9.9)$ & $129(14.8)$ & \\
\hline Mymensingh & $98(7.4)$ & $48(10.8)$ & $50(5.8)$ & \\
\hline Rajshahi & $153(11.7)$ & $34(7.6)$ & $119(13.7)$ & \\
\hline Rangpur & $126(9.6)$ & $35(7.9)$ & $91(10.4)$ & \\
\hline Sylhet & $101(7.7)$ & $39(8.8)$ & $62(7.2)$ & \\
\hline
\end{tabular}


Table 4 Prevalence (95\% confidence interval) of people with hypertension, awareness, and control

\begin{tabular}{|c|c|c|c|}
\hline Category & Hypertension & $\begin{array}{l}\text { Awareness among } \\
\text { hypertensive people }\end{array}$ & $\begin{array}{l}\text { Controlled pressure people } \\
\text { taking antihypertensive drugs }\end{array}$ \\
\hline Overall & $27.4(26.5,28.5)$ & $42.4(40.5,44.4)$ & $33.8(30.8,36.8)$ \\
\hline \multicolumn{4}{|l|}{ Age (in year) } \\
\hline 18 to 34 & $12.7(11.8,13.8)$ & $29.0(25.6,32.7)$ & $54.0(45.1,62.7)$ \\
\hline 35 to 44 & $28.4(26.6,30.3)$ & $37.4(33.3,41.7)$ & $38.3(31.5,45.6)$ \\
\hline 45 to 54 & $38.5(35.6,41.4)$ & $45.8(41.6,50.0)$ & $31.7(26.2,37.8)$ \\
\hline 55 to 64 & $46.7(44.0,49.5)$ & $53.1(49.3,56.8)$ & $33.3(27.9,39.2)$ \\
\hline 65 or more & $54.5(51.5,57.4)$ & $48.3(44.4,52.1)$ & $21.7(17.5,26.5)$ \\
\hline \multicolumn{4}{|l|}{ Gender } \\
\hline Female & $28.4(27.3,29.6)$ & $48.7(46.3,51.2)$ & $34.7(31.2,38.4)$ \\
\hline Male & $26.2(24.8,27.7)$ & $33.5(30.8,36.2)$ & $31.7(27.0,36.8)$ \\
\hline \multicolumn{4}{|l|}{ Diabetes } \\
\hline No & $25.6(24.6,26.6)$ & $39.6(37.5,41.8)$ & $34.8(31.4,38.4)$ \\
\hline Yes & $46.1(42.7,49.6)$ & $57.2(52.4,61.9)$ & $28.0(22.7,34.0)$ \\
\hline \multicolumn{4}{|l|}{ Overweight/Obese } \\
\hline No & $20.6(19.5,21.7)$ & $36.3(33.8,39)$ & $34.6(30.3,39.2)$ \\
\hline Yes & $37.1(35.6,38.7)$ & $47.5(45,50.1)$ & $33.4(29.7,37.3)$ \\
\hline \multicolumn{4}{|l|}{ Education level } \\
\hline No formal education & $35.2(33.2,37.1)$ & $43.2(40.0,46.6)$ & $27.7(23.3,32.5)$ \\
\hline Primary & $27.3(25.7,29.0)$ & $43.6(40.3,46.9)$ & $34.5(29.4,40.1)$ \\
\hline Secondary & $23.1(21.6,24.7)$ & $42.1(38.5,45.8)$ & $38.3(32.0,44.9)$ \\
\hline College or above & $22.5(20.4,24.7)$ & $38.0(33.8,42.5)$ & $42.1(33.9,50.8)$ \\
\hline \multicolumn{4}{|l|}{ Wealth quintile } \\
\hline Poorest & $23.7(21.9,25.7)$ & $32.5(28.2,37.1)$ & $32.0(24.2,41.0)$ \\
\hline Poorer & $25.2(23.1,27.3)$ & $36.4(32.3,40.7)$ & $32.8(26.2,40.2)$ \\
\hline Middle & $26.7(24.7,28.8)$ & $41.9(37.7,46.1)$ & $31.9(25.1,39.5)$ \\
\hline Richer & $28.2(26.1,30.5)$ & $46.0(42.0,50.1)$ & $38.0(31.9,44.6)$ \\
\hline Richest & $32.9(31.0,34.9)$ & $50.8(47.0,54.5)$ & $33.1(28.5,38.0)$ \\
\hline \multicolumn{4}{|l|}{ Place of residence } \\
\hline Urban & $28.5(26.8,30.2)$ & $46.6(43.3,50.0)$ & $36.0(31.3,41.0)$ \\
\hline Rural & $27.1(25.9,28.3)$ & $40.8(38.4,43.2)$ & $32.7(29.1,36.5)$ \\
\hline \multicolumn{4}{|l|}{ Division of residence } \\
\hline Dhaka & $23.6(21.4,25.9)$ & $45.4(40.5,50.5)$ & $32.9(26.2,40.5)$ \\
\hline Chattagram & $29.8(27.2,32.4)$ & $44.5(39.5,49.6)$ & $41.7(34.4,49.4)$ \\
\hline Barishal & $32.6(29.5,35.9)$ & $44.4(39.0,50.0)$ & $35.0(26.5,44.6)$ \\
\hline Khulna & $29.9(27.2,32.7)$ & $43.9(38.7,49.3)$ & $25.4(19.7,32.0)$ \\
\hline Mymensingh & $23.6(21.2,26.2)$ & $42.3(36.0,48.8)$ & $48.7(39.7,57.9)$ \\
\hline Rajshahi & $27.5(24.8,30.4)$ & $39.1(34.0,44.3)$ & $22.1(15.8,29.9)$ \\
\hline Rangpur & $30.5(28.3,32.7)$ & $33.2(28.6,38.2)$ & $27.7(20.5,36.3)$ \\
\hline Sylhet & $26.1(23.5,29.0)$ & $48.9(42.4,55.4)$ & $38.5(30.2,47.5)$ \\
\hline
\end{tabular}

$46.7 \%$ (44.0 to $49.5 \%)$ and $54.5 \%$ (51.5 to $57.4 \%)$ among people with $18-34,35-44,45-54,55-64$, and 65 or more years of age, respectively. Females had a higher prevalence $(95 \% \mathrm{CI})$ than males, $28.4 \%$ (27.3 to $29.6 \%)$ and $26.2 \%$ (24.8 to $27.7 \%)$, respectively. The prevalence also differed according to diabetes, overweight/obesity, education level, wealth status, and place and division of residence. Less than half of the people were aware about 
Table 5 Results of logistic regression analysis to investigate adjusted odds ratio (with 95\% confidence interval) for the factors associated with prevalence of hypertension, awareness among hypertensive people, and controlled hypertension among people taking antihypertensive drugs

\begin{tabular}{|c|c|c|c|c|c|c|}
\hline \multirow[t]{2}{*}{ Variable } & \multicolumn{2}{|l|}{ Prevalence } & \multicolumn{2}{|l|}{ Awareness } & \multicolumn{2}{|c|}{ Controlled hypertension } \\
\hline & AOR & $p$-value & AOR & $p$-value & AOR & $p$-value \\
\hline \multicolumn{7}{|l|}{ Age (in year) } \\
\hline 18 to 34 & Ref. (1.0) & & Ref. (1.0) & & Ref. (1.0) & \\
\hline 35 to 44 & $2.7(2.4,3.1)$ & $<0.001$ & $1.8(1.4,2.3)$ & $<0.001$ & $0.5(0.3,0.8)$ & 0.003 \\
\hline 45 to 54 & $4.5(3.9,5.2)$ & $<0.001$ & $2.8(2.1,3.6)$ & $<0.001$ & $0.4(0.3,0.6)$ & $<0.001$ \\
\hline 55 to 64 & $7.3(6.2,8.5)$ & $<0.001$ & $4.2(3.2,5.6)$ & $<0.001$ & $0.4(0.3,0.7)$ & $<0.001$ \\
\hline 65 or more & $11.9(10.1,14.1)$ & $<0.001$ & $4.2(3.2,5.6)$ & $<0.001$ & $0.3(0.2,0.5)$ & $<0.001$ \\
\hline \multicolumn{7}{|l|}{ Gender } \\
\hline Female & Ref. (1.0) & & Ref. (1.0) & & ND & \\
\hline Male & $0.8(0.7,0.8)$ & $<0.001$ & $0.4(0.3,0.5)$ & $<0.001$ & - & \\
\hline \multicolumn{7}{|l|}{ Diabetes } \\
\hline No & Ref. (1.0) & & Ref. (1.0) & & Ref. (1.0) & \\
\hline Yes & $1.7(1.5,1.9)$ & $<0.001$ & $1.6(1.3,2.0)$ & $<0.001$ & $0.7(0.5,1.0)$ & 0.059 \\
\hline \multicolumn{7}{|l|}{ Overweight/Obese } \\
\hline No & Ref. (1.0) & & Ref. (1.0) & & ND & \\
\hline Yes & $2.5(2.2,2.7)$ & $<0.001$ & $1.5(1.3,1.8)$ & $<0.001$ & - & \\
\hline \multicolumn{7}{|l|}{ Education level } \\
\hline No formal education & Ref. (1.0) & & Ref. (1.0) & & Ref. (1.0) & \\
\hline Primary & $1.1(0.9,1.2)$ & 0.38 & $1.2(1.0,1.5)$ & 0.033 & $1.1(0.8,1.5)$ & 0.74 \\
\hline Secondary & $1.0(0.9,1.2)$ & 0.59 & $1.3(1.0,1.7)$ & 0.024 & $1.2(0.8,1.7)$ & 0.38 \\
\hline College or above & $1.0(0.9,1.2)$ & 0.44 & $1.3(0.9,1.8)$ & 0.076 & $1.6(1.1,2.5)$ & 0.027 \\
\hline \multicolumn{7}{|l|}{ Wealth quintile } \\
\hline Poorest & Ref. (1.0) & & Ref. (1.0) & & ND & \\
\hline Poorer & $1.1(1.0,1.3)$ & 0.13 & $1.3(1.0,1.8)$ & 0.054 & - & \\
\hline Middle & $1.2(1.0,1.4)$ & $<0.001$ & $1.5(1.1,1.9)$ & 0.006 & - & \\
\hline Richer & $1.4(1.2,1.6)$ & $<0.001$ & $1.7(1.3,2.3)$ & $<0.001$ & - & \\
\hline Richest & $1.4(1.2,1.7)$ & $<0.001$ & $1.7(1.3,2.3)$ & $<0.001$ & - & \\
\hline \multicolumn{7}{|l|}{ Place of residence } \\
\hline Urban & $1.0(0.9,1.2)$ & 0.62 & $1.1(0.9,1.4)$ & 0.20 & ND & \\
\hline Rural & Ref. (1.0) & & Ref. (1.0) & & - & \\
\hline \multicolumn{7}{|l|}{ Division of residence } \\
\hline Dhaka & Ref. (1.0) & & Ref. (1.0) & & Ref. (1.0) & \\
\hline Chattagram & $1.5(1.2,1.8)$ & $<0.001$ & $1.0(0.7,1.4)$ & 0.85 & $1.4(0.9,2.3)$ & 0.17 \\
\hline Barisal & $1.6(1.3,2.0)$ & $<0.001$ & $1.1(0.7,1.5)$ & 0.66 & $0.9(0.6,1.6)$ & 0.80 \\
\hline Khulna & $1.3(1.1,1.6)$ & 0.003 & $1.0(0.7,1.4)$ & 0.85 & $0.8(0.5,1.2)$ & 0.27 \\
\hline Mymensingh & $1.1(0.9,1.4)$ & 0.44 & $1.0(0.7,1.4)$ & 0.90 & $1.6(0.9,2.8)$ & 0.079 \\
\hline Rajshahi & $1.4(1.2,1.7)$ & 0.001 & $0.9(0.6,1.2)$ & 0.48 & $0.6(0.3,1.0)$ & 0.061 \\
\hline Rangpur & $1.8(1.5,2.2)$ & $<0.001$ & $0.8(0.6,1.2)$ & 0.24 & $0.7(0.4,1.3)$ & 0.25 \\
\hline Sylhet & $1.4(1.1,1.7)$ & 0.006 & $1.5(1.0,2.1)$ & 0.038 & $1.2(0.7,2.0)$ & 0.44 \\
\hline
\end{tabular}

AOR Adjusted odds ratio, ND Not done, were not adjusted as the unadjusted p-values were more than 0.20

hypertensive status, $42.4 \%$ (95\% CI: 40.5 to $44.4 \%$ ). More females than males were aware of their hypertensive status, $48.7 \%$ (95\% CI: 46.3 to $51.2 \%)$ and $33.5 \%$ (95\% CI:
30.8 to $36.2 \%)$, respectively. It also differed by overweight/obesity, diabetes, and most other characteristics. About one-third of the people who were taking any 
antihypertensive drugs had controlled BP, 33.8\% (95\% CI: 30.8 to $36.8 \%)$. It decreased with age. Females had a slightly higher prevalence of controlled BP than males, 34.7\% (95\% CI: 31.2 to $38.4 \%$ ) and 31.7\% (95\% CI: 27.0 to $36.8 \%)$, respectively. It also differed by education level and people with a relatively higher education level had a higher prevalence of controlled BP than those with a lower education level.

Table 5 reports the results of multilevel logistic regression to report the factors associated with prevalence, awareness, and control of hypertension. The adjusted odds of having hypertension increased with age. The adjusted odds of hypertension among males were 0.8 (95\% CI: 0.7 to 0.8 ) times the odds among females. Similarly, diabetes (AOR: 1.7, 95\% CI: 1.5 to 1.9) and overweight/ obesity (AOR: 2.5, 95\% CI: 2.2 to 2.7 ) had significant positive association with hypertension. Factors associated with awareness of hypertension were similar as having hypertension. Older people, people with diabetes or overweight/obesity, higher education level, or wealth status had significantly higher odds of awareness about having hypertension. Lastly, older age was associated with lower odds of controlled hypertension and college or above education level was associated with higher odds of controlled hypertension.

\section{Discussion}

In this study, we observed that more than 1 in 4 people in Bangladesh may have hypertension, however, a majority of them were unaware of having it or did not have controlled hypertension despite taking antihypertensive drugs. We also identified several associated factors for these outcomes, including age, gender, having diabetes and overweight/obesity, education, wealth quintiles, and living in several divisions.

The factors that we observed in these study are similar to the factors identified by previous studies that analyzed BDHS 2011 data, however, the age group of BDHS 2017-18 (i.e., 18 years of age or older) is different than BDHS 2011 (i.e., 35 years of age or older) [3, 4, 8, 18]. Among people with $35-44,45-54,55-64$, and 65 or more years of age, the prevalence of hypertension was $17.1,25.0,30.2$, and $40.3 \%$, respectively in 2011, but the prevalence was about 1.5 times high within these age groups in our analysis, $28.4,38.5,46.7$, and $54.5 \%$, respectively [4, 8]. The awareness level and control remained similar. As mentioned, one major finding is that within less than a decade (i.e., from 2011 to 201718), among people with 35 years of age or older, the prevalence rose by more than 1.5 times, this signifies having national prevention and control programs [4]. Bangladesh and many other developing countries are currently facing double disease burden with a simultaneous higher burden of infectious and noninfectious diseases [19]. Multiple non-communicable disease programs were implemented in Bangladesh. However, those programs had inadequate planning, implementation, and monitoring to make them successful [20]. Apart from hypertension, the prevalence of many other chronic diseases (e.g., diabetes and overweight/obesity) has also increased [4]. The substantial rise in prevalence within this short period also indicates a little success of those programs. Therefore, we recommend implementing new prevention and control programs by addressing all the studied factors we identified.

We found positive association of age with prevalence and awareness of hypertension, though it was in opposite direction for hypertension control. Age is a known non-modifiable risk factor for hypertension [21]. With increasing age, structural changes take place in blood vessel walls and the risk for hypertension increases due to stiffness, sodium consumption, lack of dietary potassium, and physical inactivity [21, 22]. Older people also have increased risks for many other chronic conditions such as diabetes, overweight/obesity, chronic kidney disease, and other cardiac diseases that may impact the BP control $[3,21,23,24]$. These diseases influence the outcome of one another, share many common risk factors, and may cause more severe complications [3,21]. Overall, all age groups had lower awareness and control levels. Although we found a lower prevalence of hypertension among younger (i.e., less than 35-year-old) people, an overwhelming majority of them did not have awareness about it [4]. Having awareness and control from a relatively younger age are more beneficial to reduce future complications of hypertension [7, 21]. Moreover, adopting healthier lifestyle and dietary habits from younger age may not only reduce the future risks of it but also other chronic diseases mentioned above [21].

Gender was not associated with hypertension control, although the prevalence of hypertension was slightly lower among males, they had significantly lower awareness level. Previous studies in Bangladesh had similar findings [3, 8-10]. Though little is known about gender differences in pathophysiology of hypertension in Bangladesh, studies from other countries suggest differences in physiological and behavioral characteristics may contribute to these differences [25-28].

People with overweight/obesity or diabetes had higher odds of hypertension's prevalence and awareness. In the overall sample, about 25 and $10 \%$ people had overweight/obesity and diabetes, respectively. The overall prevalence rates of both conditions have increased in Bangladesh compared to 2011 [4, 18]. This rising trend has been observed in many other developing countries $[2,21,29,30]$. Halting the rising burden of these conditions would be important not only for reducing hypertension burden but also for reducing the complications 
arising from themselves. Mortality, morbidity, and health care costs resulting from these conditions are also high [31, 32]. Although the higher awareness among people with diabetes or overweight/obesity could appear as a welcome finding, this finding should be interpreted with caution. As people with diabetes or overweight/obesity may need more health care visits compared to people without these, that could cause this higher awareness among them [33].

We also observed differences in prevalence and awareness according to wealth quintile and place of residence. A higher prevalence of hypertension among people with higher wealth status could be due to the fact that people with a relatively higher wealth quintile in Bangladesh follow more sedentary lifestyle that could contribute to the higher prevalence [9]. The urban-rural differences in prevalence of hypertension was substantially higher in 2011 and urban residents had about 1.5 times higher prevalence than rural people [4]. However, it was minimal in 2017-18 [4]. This indicates that the prevalence is rising rapidly in rural areas compared to urban areas. However, people with a lower wealth status and rural residence have relatively lower awareness level, this difference in awareness may result from differences in health care access, availability, and utilization behavior [4]. More than two-thirds of the people in Bangladesh live in rural regions, therefore, increasing awareness in rural regions is crucial [34]. We also observed some differences in prevalence and awareness of hypertension by division of residence, this may also be due to difference in socioeconomic status and health care utilization behavior according to division of residence [3].

This study has several strengths. First, the survey had a large sample that provided us adequate power for analysis. The survey covered rural and urban regions of all administrative divisions that made the survey nationally representative. BDHS 2017-18 had a high response rate and used standard validated methods that also increased the authenticity of our results [3].

However, limitations of this study also warrant discussion. As this was a cross-sectional study, the overall association may not be causal due to uncertainty about temporal association. Although standard guidelines recommend multiple longitudinal measurements in a clinical setting $[21,35]$, the BP was measured on a single day, this may have caused some non-differential misclassification. We did not adjust for some other known risk factors (e.g., lifestyle and dietary habits) due to limitations of the dataset $[4,21]$.

\section{Conclusions}

The prevalence of hypertension has increased but awareness and control level remained lower. To reduce the future burden associated with hypertension, it is essential to address the modifiable factors associated with higher prevalence, lower awareness, and poor control of it by effective awareness and control programs. Reducing prevalence and increasing control of hypertension among older people and males are important. Reducing prevalence of overweight/obesity and diabetes may reduce future burden of hypertension, as well as complications arising from it. Increasing awareness among younger people and prioritizing rural regions to raise awareness is essential.

\section{Abbreviations \\ AOR: Adjusted odds ratio; BP: Blood pressure; BDHS: Bangladesh \\ Demographic and Health Survey; BMI: Body mass index; DBP: Diastolic blood pressure; LMIC: Low- and middle-income countries; SBP: Systolic blood pressure; SE: Standard errors; UOR: Unadjusted odds ratio}

\section{Supplementary Information}

The online version contains supplementary material available at https://doi. org/10.1186/s40885-021-00174-2.

Additional file 1: Supplemental Table 1. Prevalence ( $95 \%$ confidence interval) of people with hypertension, awareness, and control among 230-year-old people.

Additional file 2: Supplemental Table 2. Results of logistic regression analysis to investigate unadjusted odds ratio (with 95\% confidence interval) for the factors associated with prevalence of hypertension, awareness among hypertensive people, and controlled hypertension among people taking antihypertensive drugs.

\section{Acknowledgements}

The authors are thankful to the ICF International, Rockville, Maryland, USA for giving the permission to use the data.

\section{Authors' contributions}

GMAK and RDJ did the literature review and conceptualized the study, GMAK and JN prepared the first draft of the manuscript, GMAK performed statistical analyses; RDG and JN did the critical review of the manuscript. All authors read and approved the final manuscript.

Authors' information

No additional information to disclose.

Funding

No financial support was received for this study.

Availability of data and materials

Data may be made available upon request to the ICF International, Maryland, USA.

\section{Declarations}

Ethics approval and consent to participate

Secondary data from BDHS 2011 were analyzed for this study and the survey data was anonymized. The Institutional Review Boards (IRBs) of the ICF International, Rockville, Maryland, USA and Bangladesh Medical Research Council, Dhaka, Bangladesh approved the BDHS 2017-18 protocol. This dataset was available for scientific and academic use upon approval from the ICF International; the permission for using the data was obtained in December 2020. All participants provided informed consent to participate into the survey.

Consent for publication

Not applicable. 


\section{Competing interests}

The authors declare that they have no competing interests.

\section{Author details}

'Department of Epidemiology and Public Health, University of Maryland School of Medicine, Baltimore, MD 21201, USA. ${ }^{2}$ Department of Epidemiology and Biostatistics, Arnold School of Public Health, University of South Carolina, Columbia, SC 29208, USA. ${ }^{3}$ Chattagram International Dental College and Hospital, Chittagong, Bangladesh.

Received: 21 January 2021 Accepted: 27 June 2021

Published online: 01 September 2021

\section{References}

1. Forouzanfar MH, Liu P, Roth GA, Ng M, Biryukov S, Marczak L, et al. Global burden of hypertension and systolic blood pressure of at least 110 to $115 \mathrm{~mm} \mathrm{hg}$, 19902015. JAMA. 2017;317(2):165-82. https://doi.org/10.1001/jama.2016.19043.

2. Zhou B, Bentham J, Di Cesare M, Bixby H, Danaei G, Cowan MJ, et al. Worldwide trends in blood pressure from 1975 to 2015: a pooled analysis of 1479 population-based measurement studies with $19 \cdot 1$ million participants. Lancet. 2017;389(10064):37-55. https://doi.org/10.101 6/S0140-6736(16)31919-5.

3. Kibria GMA, Swasey K, Hasan MZ, Choudhury A, Gupta RD, Abariga SA, et al. Determinants of hypertension among adults in Bangladesh as per the JNC7 and 2017 ACC/AHA hypertension guidelines. J Am Soc Hypertens. 2018(11): e45-55. https://doi.org/10.1016/j.jash.2018.10.004.

4. National Institute of Population Research and Training (NIPORT), Mitra and Associates, ICF International. Bangladesh Demographic and Health Survey 2017-18. Dhaka; 2020. https://dhsprogram.com/pubs/pdf/fr265/fr265.pdf. Accessed 1 Jan 2021

5. National Institute of Population Research and Training (NIPORT), Mitra and Associates, ICF International. Bangladesh Demographic and Health Survey 2011. Dhaka; 2013. https://dhsprogram.com/pubs/pdf/fr265/fr265.pdf. Accessed 20 Jun 2018

6. Islam AKMM, Majumder AAS. Hypertension in Bangladesh: a review. Indian Heart J. 2012;64(3):319-23. https://doi.org/10.1016/S0019-4832(12)60096-0.

7. Alam DS, Chowdhury MAH, Siddiquee AT, Ahmed S, Niessen LW. Awareness and control of hypertension in Bangladesh: follow-up of a hypertensive cohort. BMJ Open. 2014;4(12):e004983. https://doi.org/10.1136/bmjopen-2014-004983.

8. Chowdhury MAB, Uddin MJ, Haque MR. Ibrahimou B. hypertension among adults in Bangladesh: evidence from a national cross-sectional survey. BMC Cardiovasc Disord. 2016;16(1):22. https://doi.org/10.1186/s1 2872-016-0197-3.

9. Kibria GMA, Swasey K, Das Gupta R, Choudhury A, Nayeem J, Sharmeen A, et al. Differences in prevalence and determinants of hypertension according to rural-urban place of residence among adults in Bangladesh. J Biosoc Sci. 2019;51(4):578-90. https://doi.org/10.1017/S0021932018000366.

10. Harshfield E, Chowdhury R, Harhay MN, Bergquist H, Harhay MO. Association of hypertension and hyperglycaemia with socioeconomic contexts in resource-poor settings: the Bangladesh demographic and health survey. Int J Epidemiol. 2015;44(5):1625-36. https://doi.org/10.1093/ije/dyv087.

11. Rahman M, Williams G, Al MA. Gender differences in hypertension awareness, antihypertensive use and blood pressure control in Bangladeshi adults: findings from a national cross-sectional survey. J Health Popul Nutr. 2017;36(1):23. https://doi.org/10.1186/s41043-017-0101-5.

12. Chalmers J, MacMahon S, Mancia G, Whitworth J, Beilin L, Hansson L, et al. 1999 World Health Organization-International Society of Hypertension Guidelines for the management of hypertension. Guidelines sub-committee of the World Health Organization. Clin Exp Hypertens. 1999;21:1009-60.

13. Khosla T, Lowe CR. Indices of obesity derived from body weight and height. Br J Prev Soc Med. 1967;21:122-8.

14. WHO Expert Consultation. Appropriate body-mass index for Asian populations and its implications for policy and intervention strategies. Lancet Lond Engl. 2004;363:157-63.

15. Joliffe $I T$, Morgan BJ. Principal component analysis and exploratory factor analysis. Stat Methods Med Res. 1992; (1):69-95. https:/doi.org/10.1177/096228029200100105.

16. Maldonado G, Greenland S. Simulation study of confounder-selection strategies. Am J Epidemiol. 1993;138(11):923-36. https://doi.org/10.1093/ oxfordjournals.aje.a116813.

17. Stata Corporation, College Station, Texas. StataCorp. 2017. https://www.stata. com/support/faqs/resources/citing-software-documentation-fags/. Accessed 8 May 2017
18. Kibria GMA, Hashan MR, Hossain MM, Zaman SB, Stennett CA. Clustering of hypertension, diabetes and overweight/obesity according to socioeconomic status among Bangladeshi adults. J Biosoc Sci. 2021;53(2):157-66. https://doi. org/10.1017/S0021932020000085.

19. Bygbjerg IC. Double burden of noncommunicable and infectious diseases in developing countries. Science. 2012;337(6101):1499-501. https://doi.org/1 $0.1126 /$ science. 1223466.

20. Biswas T, Pervin S, Tanim MIA, Niessen L, Islam A. Bangladesh policy on prevention and control of non-communicable diseases: a policy analysis. BMC Public Health. 2017;17(1):582. https://doi.org/10.1186/s12 889-017-4494-2.

21. Oparil S, Acelajado MC, Bakris GL, Berlowitz DR, Cífková R, Dominiczak AF, et al. Hypertension. Nat Rev Dis Primer. 2018;4(1):18014. https://doi.org/10.1 038/nrdp.2018.14.

22. Pinto E. Blood pressure and ageing. Postgrad Med J. 2007;83(976):109-14. https://doi.org/10.1136/pgmj.2006.048371.

23. Chowdhury MAB, Uddin MJ, Khan HMR, Haque MR. Type 2 diabetes and its correlates among adults in Bangladesh: a population based study. BMC Public Health. 2015;15(1):1070. https://doi.org/10.1186/s12889-015-2413-y.

24. Akter J, Shahjahan M, Hossain S, Chowdhury H, Ahmed K, Fatema K, et al. Determinants of overweight and obesity among Bangladeshi diabetic women of reproductive age. BMC Res Notes. 2014;7(1):513. https://doi.org/1 0.1186/1756-0500-7-513.

25. Agho KE, Osuagwu UL, Ezeh OK, Ghimire PR, Chitekwe S, Ogbo FA. Gender differences in factors associated with prehypertension and hypertension in Nepal: a nationwide survey. PLoS One. 2018;13(9):e0203278. https://doi. org/10.1371/journal.pone.0203278.

26. Barker M, Chorghade G, Crozier S, Leary S, Fall C. Gender differences in body mass index in rural India are determined by socio-economic factors and lifestyle. J Nutr. 2006;136(12):3062-8. https://doi.org/10.1093/ jn/136.12.3062.

27. Everett B, Zajacova A. Gender differences in hypertension and hypertension awareness among young adults. Biodemography Soc Biol. 2015;61(1):1-17. https://doi.org/10.1080/19485565.2014.929488.

28. Doumas M, Papademetriou V, Faselis C, Kokkinos P. Gender differences in hypertension: myths and reality. Curr Hypertens Rep. 2013;15(4):321-30. https://doi.org/10.1007/s11906-013-0359-y.

29. NCD Risk Factor Collaboration (NCD-RisC). Worldwide trends in body-mass index, underweight, overweight, and obesity from 1975 to 2016: a pooled analysis of 2416 population-based measurement studies in 128.9 million children, adolescents, and adults. Lancet Lond Engl. 2017;390:2627-42.

30. Menke A, Casagrande S, Geiss L, Cowie CC. Prevalence of and trends in diabetes among adults in the United States, 1988-2012. JAMA. 2015;314(10): 1021. https://doi.org/10.1001/jama.2015.10029.

31. Berrington de Gonzalez A, Hartge P, Cerhan JR, Flint AJ, Hannan L, RJ MI, et al. Body-Mass Index and Mortality among 1.46 Million White Adults. N Engl J Med. 2010;363:2211-9.

32. Wang $\mathrm{H}$, Naghavi M, Allen C, Barber RM, Bhutta ZA, Carter A, et al. Global, regional, and national life expectancy, all-cause mortality, and cause-specific mortality for 249 causes of death, 1980-2015: a systematic analysis for the global burden of disease study 2015. Lancet. 2016;388(10053):1459-544. https://doi.org/10.1016/S0140-6736(16)31012-1.

33. Finley CR, Chan DS, Garrison S, Korownyk C, Kolber MR, Campbell S, et al. What are the most common conditions in primary care? Systematic review. Can Fam Physician Med Fam Can. 2018;64:832-40.

34. Bureau of Statistics, Ministry of Planning, Government of Bangladesh. Bangladesh Statistics 2017. 2017. http://bbs.portal.gov.bd/sites/default/files/ files/bbs.portal.gov.bd/page/a1d32f13_8553_44f1_92e6_8ff80a4ff82e/Bangla desh\%20\%20Statistics-2017.pdf. Accessed 16 Aug 2019.

35. Whelton PK, Carey RM, Aronow WS, Casey DE, Collins KJ, Dennison Himmelfarb C, et al. 2017 ACC/AHA/AAPA/ABC/ACPM/AGS/APhA/ASH/ASPC/NMA/PCNA guideline for the prevention, detection, evaluation, and Management of High Blood Pressure in adults: a report of the American College of Cardiology/ American Heart Association task force on clinical practice guidelines. Hypertension. 2018:HYP.0000000000000065.

\section{Publisher's Note}

Springer Nature remains neutral with regard to jurisdictional claims in published maps and institutional affiliations. 